\title{
Electrical Capacitance Tomography Measurement of the Migration of Ice Frontal Surface in Freezing Soil
}

\author{
J. Liu ${ }^{1,2}$, X.M. Suo ${ }^{1}$, S.S. Zhou ${ }^{1}$, S.Q. Meng ${ }^{1}$, S.S. Chen ${ }^{1}$, H.P. Mu $^{3}$ \\ ${ }^{1}$ School of Civil Engineering, Henan Polytechnic University, 45400, Jiaozuo, China, liujinghpu@163.com, (J. Liu) \\ ${ }^{2}$ Key Laboratory of Efficient Utilization of Low and Medium Grade Energy (Tianjin University), Ministry of Education of \\ China, 300072, Tianjin, China \\ ${ }^{3}$ School of Energy, Power and Mechanical Engineering, North China Electric Power University, 102206, Beijing, China, \\ woodengoal@126.com, (H.P.Mu)
}

\begin{abstract}
The tracking of the migration of ice frontal surface is crucial for the understanding of the underlying physical mechanisms in freezing soil. Owing to the distinct advantages, including non-invasive sensing, high safety, low cost and high data acquisition speed, the electrical capacitance tomography (ECT) is considered to be a promising visualization measurement method. In this paper, the ECT method is used to visualize the migration of ice frontal surface in freezing soil. With the main motivation of the improvement of imaging quality, a loss function with multiple regularizers that incorporate the prior formation related to the imaging objects is proposed to cast the ECT image reconstruction task into an optimization problem. An iteration scheme that integrates the superiority of the split Bregman iteration (SBI) method is developed for searching for the optimal solution of the proposed loss function. An unclosed electrodes sensor is designed for satisfying the requirements of practical measurements. An experimental system of one dimensional freezing in frozen soil is constructed, and the ice frontal surface migration in the freezing process of the wet soil sample containing five percent of moisture is measured. The visualization measurement results validate the feasibility and effectiveness of the ECT visualization method.
\end{abstract}

Keywords: Freezing soil, ice frontal surface migration, electrical capacitance tomography, visualization measurement, image reconstruction method.

\section{INTRODUCTION}

The frost heave and thaw settlement of the frozen soil endanger the safety of various frozen soil engineering applications, and thus numerous investigations on the underlying physical mechanisms and the frozen damage prevention have been done to prevent catastrophic effect [1], [2]. In the process of freezing and thawing, each component of the frozen soil is in a dynamic equilibrium, various external conditions will bring the variations of the components inside the frozen soil and the migration of the freezing interface, and thus understanding the underlying physical mechanisms of the changes of the components and the freezing interface movement is crucial in geocryology [3]. Currently, various measurement techniques, e.g., CT techniques [4], [5], geological radar [6], electric resistance measuring method [7], and portable nuclear magnetic resonance detector [8], etc., are available for the measurements of the component distributions of frozen soil and the tracking of the freezing interface movement. In [9], the authors observed the component distributions of the frozen soil section in the process of freezing, frozen, and the location of the freezing interface by scanning electron microscope. However, the method will damage the freeze status of the frozen soil in the process of measurement; furthermore, it is also hard to achieve the online and nonintrusive measurement on the freezing interface of the frozen soil. Generally, owing to the complexities and characteristics of the problem, seeking a reliable method to measure the component distributions and the tracking of the freezing interface remains a critical problem.

As a promising noninvasive visualization measurement method, the ECT technique attempts to reconstruct the permittivity distributions of the imaging objects by means of an appropriate algorithm from the given capacitance measurement data, in which reconstructing high-quality images is crucial for practical engineering applications. The ECT image reconstruction process holds two key phases, i.e., the forward problem and the inverse problem. The former tries to compute the capacitance values from the known permittivity distributions, and it is a well-posed problem. On the contrary, the latter tries to estimate the permittivity distribution from the given capacitance data, and it is an illposed problem. Owing to the distinct advantages, e.g., noninvasive sensing, high safety, low cost, easy 
implementation, and high temporal resolution, the ECT visualization measurement method has been successfully applied to various industrial processes for the monitoring of the objects of interest. In contrast to common measurement methods, naturally, in this paper we use the ECT method to achieve the visualization measurement of the freezing process, and the main contributions are outlined as follows.

1) For the first time, the ECT visualization measurement method is introduced to visualize the freezing process in the frozen soil.

2) As opposed to common ECT sensors, an unclosed electrodes ECT sensor is designed to satisfy the measurement requirements of the freezing process in the frozen soil.

3) A loss function with multiple regularizers that integrate the prior knowledge associated with the imaging objects is proposed to convert the ECT image reconstruction task into an optimization problem via the framework of the Tikhonov regularization method. An iteration scheme that integrates the advantages of the SBI method is developed for seeking the optimal solution of the proposed loss function.

4) Experimental studies are implemented to measure the freezing interface movement inside frozen soil and the feasibility and effectiveness of the ECT visualization measurement method are validated. As a result, a promising approach is introduced to investigate the underlying physical mechanisms of the freezing process.

The rest of this paper is organized as follows. In Section 2, the ECT measurement principle is concisely introduced. In Section 3, an imaging method is proposed for the ECT image reconstruction. Section 4 describes the experimental system. In Section 5, we describe the measurement procedures in detail. The experimental results and discussions are demonstrated in Section 6. Finally, we summarize the main conclusions in Section 7.

\section{ECT PRINCIPLES}

\section{A. ECT model}

In real-world applications, the electric field inside an ECT sensor can be formulated as [10], [11]:

$$
\nabla \cdot[\varepsilon(x, y) \nabla \phi(x, y)]=0
$$

where $\varepsilon(x, y)$ and $\phi(x, y)$ are the permittivity and electrical potential distributions, respectively. The relationship of the capacitance and the permittivity distribution can be written as [12]:

$$
C=\frac{Q}{V}=-\frac{1}{V} \iint_{\Gamma} \varepsilon(x, y) \nabla \phi(x, y) d \Gamma
$$

where $Q$ is the electric charge; $V$ indicates the potential difference between two electrodes forming the capacitance and $\Gamma$ is the electrode surface. Finally, we can approximate (2) as follows [12]:

$$
S x=C+r
$$

where $\boldsymbol{S}$ represents a normalized matrix of dimension $m \times n ; \boldsymbol{x}$ stands for an $n \times 1$ dimensional unknown vector; $\boldsymbol{C}$ is an $m \times 1$ vector, stands for a normalized capacitance vector; $\boldsymbol{r}$ is an $m \times 1$ dimensional noise vector.

It is necessary to mention that equation (3) is a linearization model that has found wide applications in the field of the ECT measurement, and can be modified according to a specific imaging object. Currently, researchers had proposed different models to improve the imaging quality, e.g., the nonlinear model, the models that simultaneously consider the inaccurate properties of the reconstruction model and the measurement data, etc., and more details can be found in [17], [19], [20]. In this paper, for the sake of easy computation and satisfying the demands of real-world applications, the linearization model is employed in practical measurements. In the future, more investigations on the imaging model and its influence on the imaging quality should be further conducted.

\section{B. Common image reconstruction methods}

Acquiring high-quality images plays an important role in the understanding of the underlying physical mechanisms of the imaging objects, and thus numerous algorithms have been developed for the ECT imaging tasks. For example, the linear back-projection method [10], the standard Tikhonov regularization method [13], the Landweber iteration algorithm [14], the offline iteration and online reconstruction algorithm [15], the generalized vector sampled pattern matching method [16], the generalized Tikhonov regularization techniques [17]-[20], the simulated annealing method [21], and other imaging methods can be traced back to [22]-[28]. Owing to the ill-posedness of the ECT imaging problem, seeking a fast and stable numerical method to ensure a high-quality imaging result remains an open problem.

\section{IMAGE RECONSTRUCTION METHOD}

Owing to ill-posedness, (3) is often converted into an optimization of a loss function consisting of the data fidelity and the regularizers that incorporate the prior knowledge related to the properties of the underlying imaging objects by introducing the Tikhonov regularization method to ensure a stable inversion solution. In order to improve the imaging quality, in this paper a new loss function is proposed to model the ECT imaging problem, which can be formulated as:

$$
\min \left\{\frac{1}{2}\|\boldsymbol{S} \boldsymbol{x}-\boldsymbol{C}\|^{2}+\frac{\alpha_{1}}{2}\|\boldsymbol{W} \boldsymbol{x}\|^{2}+\alpha_{2}\|\boldsymbol{X}\|_{*}+\alpha_{3} \operatorname{TV}(\boldsymbol{x})\right\}
$$

where $\alpha_{j}>0 \quad(j=1,2,3)$ are the user-determined regularization parameters; operator $\|\cdot\|_{*}$ defines the nuclear norm for a matrix, and it can be specified as 
$\|\boldsymbol{X}\|_{*}=\sum_{k} \sigma_{k}$, in which $\sigma_{k}$ is the singular value of matrix $\boldsymbol{X}$ that is formed by reshaping vector $\boldsymbol{x} ; \operatorname{TV}(\boldsymbol{x})$ represents the total variation operator, and for a discrete image it can be defined as [29]:

$$
\mathrm{TV}(\boldsymbol{x})=\left\|\mathrm{D}_{x} \boldsymbol{x}\right\|_{1}+\left\|\mathrm{D}_{y} \boldsymbol{x}\right\|_{1}
$$

where $\mathrm{D}_{x}$ and $\mathrm{D}_{y}$ represent the first-order difference operators along the horizontal and vertical directions of a two-dimensional image, respectively.

Finally, a new loss function for the ECT image reconstruction problem can be specified as:

$$
\min \left\{\frac{1}{2}\left\|S_{x}-\boldsymbol{C}\right\|^{2}+\alpha_{1}\left\|W_{X}\right\|^{2}+\alpha_{2}\|X\|_{.}+\alpha_{3}\left\|\mathrm{D}_{x} x\right\|_{1}+\alpha_{4}\left\|\mathrm{D}_{y} x\right\|_{1}\right\}
$$

According to the optimization theory, (6) can be rewritten as:

$$
\left\{\begin{array}{l}
\min \left\{\frac{1}{2}\|\boldsymbol{S} \boldsymbol{x}-\boldsymbol{C}\|^{2}+\alpha_{1}\|\boldsymbol{W} \boldsymbol{x}\|^{2}+\alpha_{2}\|\boldsymbol{X}\|_{.}+\alpha_{3}\left\|_{\boldsymbol{d}_{1}}\right\|_{1}+\alpha_{4}\left\|_{\boldsymbol{d}_{2}}\right\|_{1}\right\} \\
\text { s.t. } \boldsymbol{d}_{1}=\mathrm{D}_{x} \boldsymbol{x}, \boldsymbol{d}_{2}=\mathrm{D}_{y} \boldsymbol{x}
\end{array}\right.
$$

Applying the SBI method to (7) yields [30]:

$$
\begin{gathered}
\min \left\{\begin{array}{c}
\frac{1}{2}\left\|\boldsymbol{S}_{\boldsymbol{x}}-\boldsymbol{C}\right\|+\alpha_{1}\|\boldsymbol{W} \boldsymbol{x}\|+\alpha_{2}\|\boldsymbol{x}\|_{*}+\alpha_{3}\left\|\boldsymbol{d}_{1}\right\|_{1}+\alpha_{4}\left\|\boldsymbol{d}_{2}\right\|_{1} \\
+\frac{\mu_{1}}{2}\left\|\boldsymbol{d}_{1}-\mathrm{D}_{x} \boldsymbol{x}-\boldsymbol{b}_{1}^{k}\right\|^{2}+\frac{\mu_{2}}{2}\left\|\boldsymbol{d}_{2}-\mathrm{D}_{y} \boldsymbol{x}-\boldsymbol{b}_{2}^{k}\right\|^{2}
\end{array}\right\} \\
\boldsymbol{b}_{1}^{k+1}=\boldsymbol{b}_{1}^{k}+\mathrm{D}_{x} \boldsymbol{x}^{k+1}-\boldsymbol{d}_{1}^{k+1} \\
\boldsymbol{b}_{2}^{k+1}=\boldsymbol{b}_{2}^{k}+\mathrm{D}_{y} \boldsymbol{x}^{k+1}-\boldsymbol{d}_{2}^{k+1}
\end{gathered}
$$

For the sake of easy computation, following the computational strategies presented in [31], we can decouple (8) as the following sub-optimization problems:

$$
\begin{gathered}
\boldsymbol{d}_{1}^{k}=\min \left\{\alpha_{3}\left\|\boldsymbol{d}_{1}\right\|_{1}+\frac{\mu_{1}}{2}\left\|\boldsymbol{d}_{1}-\mathrm{D}_{x} \boldsymbol{x}^{k}-\boldsymbol{b}_{1}^{k}\right\|^{2}\right\} \\
\boldsymbol{d}_{2}^{k+1}=\min \left\{\alpha_{4}\left\|\boldsymbol{d}_{2}\right\|_{1}+\frac{\mu_{2}}{2}\left\|\boldsymbol{d}_{2}-\mathrm{D}_{y} \boldsymbol{x}^{k}-\boldsymbol{b}_{2}^{k}\right\|^{2}\right\} \\
\boldsymbol{x}^{k+1}=\min \left\{\begin{array}{l}
\frac{1}{2}\|\boldsymbol{S} \boldsymbol{x}-\boldsymbol{C}\|^{2}+\alpha_{1}\|\boldsymbol{W} \boldsymbol{x}\|^{2}+\alpha_{2}\|\boldsymbol{x}\|_{*}+ \\
\frac{\mu_{1}}{2}\left\|\boldsymbol{d}_{1}^{k+1}-\mathrm{D}_{x} \boldsymbol{x}-\boldsymbol{b}_{1}^{k}\right\|^{2}+\frac{\mu_{2}}{2}\left\|\boldsymbol{d}_{2}^{k+1}-\mathrm{D}_{y} \boldsymbol{x}-\boldsymbol{b}_{2}^{k}\right\|^{2}
\end{array}\right\}
\end{gathered}
$$
can be concisely called as the multiple regularizer reconstruction (MRR) method can be developed for solving (6), which can be outlined as follows:

Step 1. Specify the algorithmic parameters and the initial values.

Step 2. Update variable $\boldsymbol{d}_{1}^{k+1}$ by solving (11) using the shrinkage algorithm.

Step 3. Update variable $\boldsymbol{d}_{2}^{k+1}$ by solving (12) using the shrinkage algorithm.

Step 4. Update variable $\boldsymbol{X}^{k+1}$ by solving (13) using the IST method [32] and the singular value thresholding (SVT) algorithm [33].

Step 5. Update variable $\boldsymbol{b}_{1}^{k+1}$ according to (9).

Step 6. Update variable $\boldsymbol{b}_{2}^{k+1}$ according to (10).

Step 7. Loop to Step 2 until a predetermined iteration stopping criterion is satisfied.

Additionally, it is worth to note that the inversion solution is in a range $\left[\varpi_{1}, \varpi_{2}\right]$, and thus a projected operator is introduced to the iterative scheme:

$$
\boldsymbol{x}^{k+1}=\operatorname{Project}\left\{\boldsymbol{x}^{k+1}\right\}
$$

where,

$$
\operatorname{Project}\left[\mathbf{z}_{j}\right]= \begin{cases}\varpi_{1}, & \mathbf{z}_{j}<\varpi_{1} \\ \mathbf{z}_{i, j}, & \varpi_{1} \leq \mathbf{z}_{j} \leq \varpi_{2} \\ \varpi_{2}, & \mathbf{z}_{j}>\varpi_{2}\end{cases}
$$

\section{EXPERIMENTAL SYSTEMS}

\section{A. Experimental platform}

According to the Program of the Artificial Frozen Stratigraphic Classification and Laboratory Test, the temperature gradient in the soil column must be made to bring out one dimensional freeze along upward direction of the soil column. Experimental system for the freezing of frozen soil is shown in Fig.1., which contains three units, such as the cooling unit, the low temperature circulation shower unit, and the frozen soil measurement unit. The minimum temperature of the system refrigerating fluid (the concentration of glycol is 50 percent) in the experiment is 23.6 degrees Celsius, the thermostatic control in different low temperatures of the refrigerating fluid can be achieved by the temperature controller. One dimensional freezing under different conditions of the frozen soil samples can be achieved by the refrigerating fluid's circulation at the roof and bottom channel. 


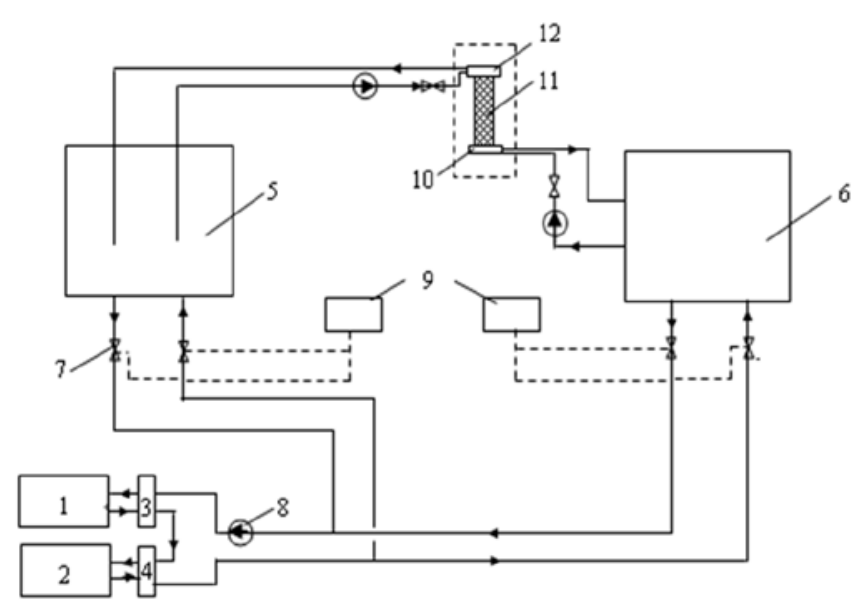

Fig.1. Experimental platform. (1. Refrigerator a; 2. Refrigerator b; 3 . Plate type exchanger a; 4 . Plate type exchanger b; 5 . Cold shower box a; 6 . Cold shower box b; 7. Electromagnetic valve; 8 . Recycle pump; 9. PID temperature controller; 10. Bottom; 11. Frozen soil sample; 12 . Roof.).

\section{B. ECT system}

The ECT system used in the research experiment has three components, e.g., a multi-electrode sensor, the sensing electronics and a computer. A set of electrodes is mounted around the measurement object and the capacitance values between all single electrode combinations are measured. The sensing electronics achieve the data acquisition. The computer controls the system hardware and implements image reconstruction and data analysis.

Different from common ECT sensors, a new unclosed electrodes sensor is designed to implement the visualization measurement of the freezing process, which is shown in Fig.2. and Fig.3. In this experiment, the width, $a$, and the length, $b$, of the measurement zone are 60 and $80 \mathrm{~mm}$, respectively. The number of the electrodes is 10 and the width of the electrode $l$ is $14 \mathrm{~mm}$.

The capacitance acquisition system is based on the AC based charging and discharging circuit and the main principles can be found in [34]. The capacitance measurement circuit has a high sensitivity, and can measure the capacitance value $0.01 \mathrm{pF}$.

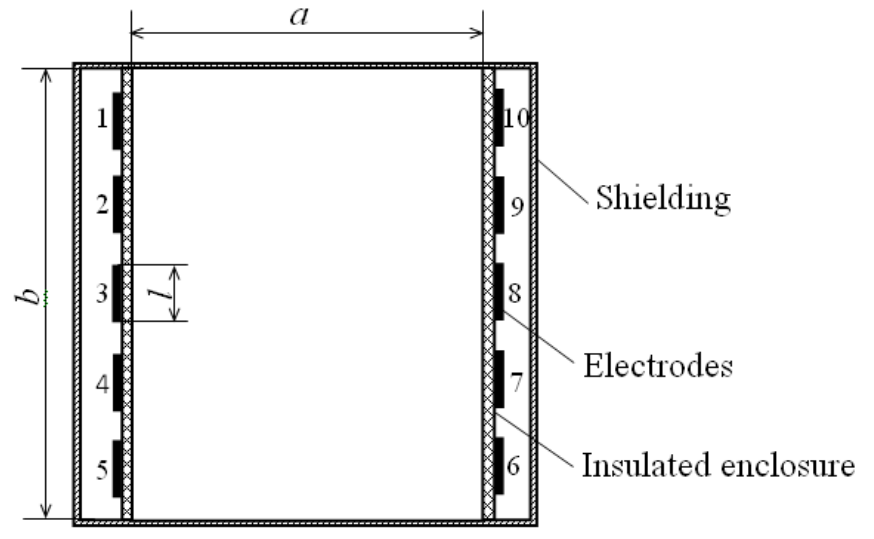

Fig.2. Arrangement of the unclosed electrodes sensor.

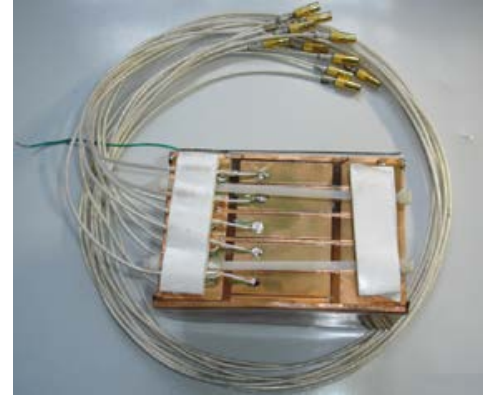

Fig.3. Unclosed electrodes sensor.

\section{MEASUREMENT PROCEDURE}

\section{A. Sample preparation}

After setting the soil sample into a bake oven to dry it, take it out and pulverize it, then mix it to a wet sample with the moisture of $5 \%$. The wet sample is placed in airtight glass container for 24 hours to ensure the soil moisture to be homogeneous.

\section{B. Measurement of the starting freezing temperature}

Take the prepared sample into the test-tube and put the test-tube into the refrigerating fluid, along with gradually decreasing temperature of the refrigerating fluid the temperature drop curve is obtained by the copper-kang copper thermocouple sensor placed in the center of the soil sample, and the starting freezing temperature of the wet soil sample is -0.42 degrees Celsius.

\section{ECT measurement}

According to the above discussions, the procedure of the measurement of the migration of ice frontal surface can be summarized as follows.

Step 1. The calibration of the ECT system. The ECT system is calibrated using high and low medium. In this experiment, the low calibration medium uses the dry soil sample and the high calibration medium employs the wet soil sample with the moisture of $5 \%$.

Step 2. The wet soil sample is fixed in the freezing device. The unclosed sensor filled with the wet soil sample is set at the location between the roof and the freeze board of the frozen soil measurement system, and the sensor is covered by the insulating layer with a thickness of $60 \mathrm{~mm}$.

Step 3. The freezing of the wet soil sample. Open the refrigerator and the circulating pump, and the cryogenic fluid flows through the roof; finally, the one-dimensional freezing of the wet soil samples is achieved.

Step 4. The ECT visualization measurement. In this process, we use the ECT method to measure the distributions of the medium, and the MRR method is used to implement the image reconstruction.

In this paper, all images are visualized using $39 \times 29$ pixels. It is necessary to mention that the reconstruction domain can be divided into different pixels. In practical ECT measurements, the number of pixels is equivalent to the number of the unknown variables. Owing to the limits of the sensor structure, the number of the known measurement data 
(i.e., the independent capacitance measurement data) is often smaller than that of the unknown variables, which leads to the difficulties of the numerical computation. When the number of the pixels is large, the reconstruction domain can be well approximated, which will aggravate the underdetermined property of the image reconstruction problem and the difficulty of the numerical computation. On the contrary, when the number of the pixels is small, the underdetermined property of the image reconstruction problem can be mitigated, but the approximation of the reconstruction domain may be unsatisfactory, which will influence the recognition of the reconstruction objects. Generally, the number of the pixels is experientially determined in real-world applications. The issue should be further investigated in the future.

\section{MeAsurement RESULTS AND DISCUSSIONS}

In the measurement, open the recycle pump when the temperature of the refrigerating fluid decreases to 0 degrees Celsius, then the refrigerating fluid flowing over the roof and the temperature will be collected. The measurement time instants in which each station temperature decreases to the starting freeze temperature ( -0.42 degrees Celsius) are 2.1 hours, 3.3 hours, 3.8 hours, 5.5 hours and 5.9 hours, respectively. In the MRR method, the algorithmic parameters are $\alpha_{1}=0.08, \alpha_{2}=0.08, \alpha_{3}=0.01$, $\alpha_{4}=0.01$, and the number of iterations is 5 . Fig.4. to Fig.8. present the images reconstructed by the MRR method when $\mathrm{t}=0,3.3,3.8,5.5,5.9$ hours, respectively.

Fig.4. to Fig.8. show the images reconstructed by the MRR method. It can be found that in the process of freezing, the wet soil sample presents different zones with different gray level values, and the location of the low gray value zones is higher than that of the high gray value zones, and the surface between different zones can be obviously identified. One of the possible reasons is that the permittivity of water is between 78.5 and 90.6, the permittivity of ice is about 3 under the corresponding experimental conditions. Owing to the large permittivity value of water, the permittivity will obviously decrease when the moisture of the wet soil sample is frozen. Therefore, in the process of freezing, the permittivity of the top frozen zone is smaller than that of the bottom zone. Especially, it can be seen from Fig.6. that zones I and II are the frozen and unfrozen domains, respectively, and the ice frontal surface between both zones can be obviously observed. Following the above discussions, we can conclude that the ECT method is successful in the visualization measurement of the freezing process.

A high-quality image will be helpful for the understanding of the complicated mechanisms of the freezing process. Studies indicate that the reconstruction quality of the ECT method is influenced by many factors, e.g., the quality of the measured capacitance signal, the sensor structure, the calibration method, the imaging model, the reconstruction method, the size of the measurement domain, the permittivity distribution of the imaging objects, the difference of the permittivity of the measurement objects, the location of the measurement objects, etc. In other words, to enable the ECT visualization method to be applied to real-world environment, it is vital to further improve the hardware and software of the ECT system in the future.

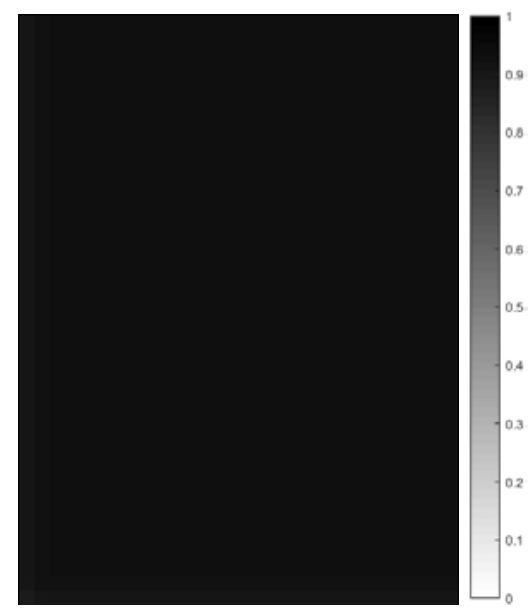

Fig.4. The image reconstructed by the MRR method when $t=0$.

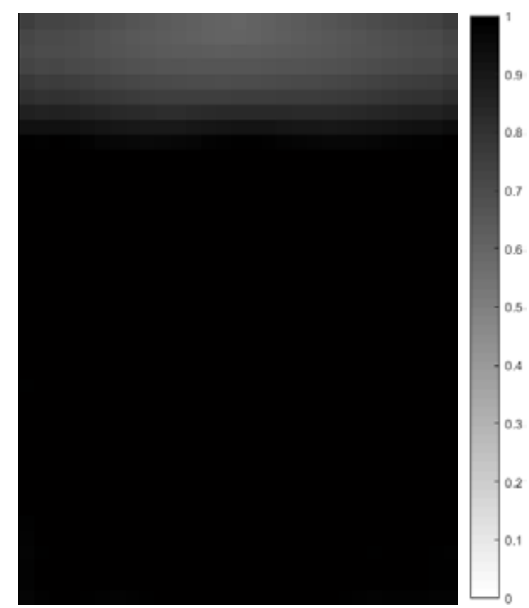

Fig.5. The image reconstructed by the MRR method when $\mathrm{t}=3.3 \mathrm{~h}$.

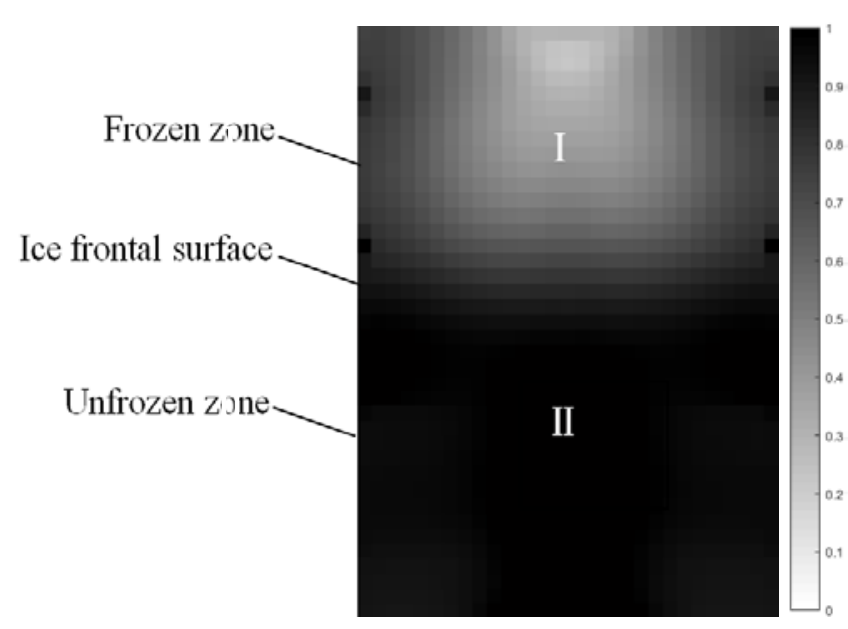

Fig.6. The image reconstructed by the MRR method when $\mathrm{t}=3.8 \mathrm{~h}$ 


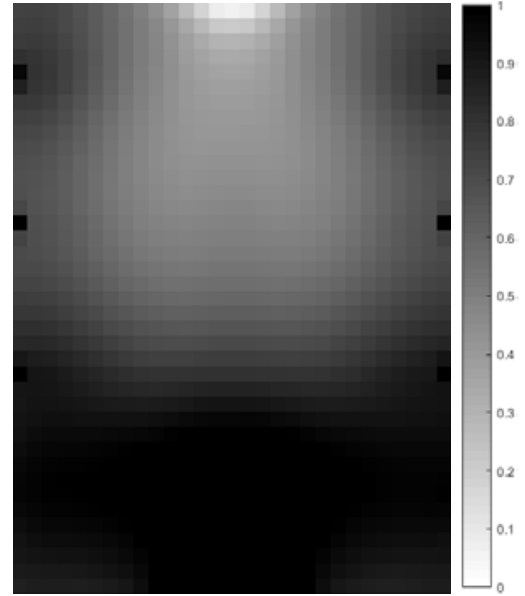

Fig.7. The image reconstructed by the MRR method when $\mathrm{t}=5.5 \mathrm{~h}$.

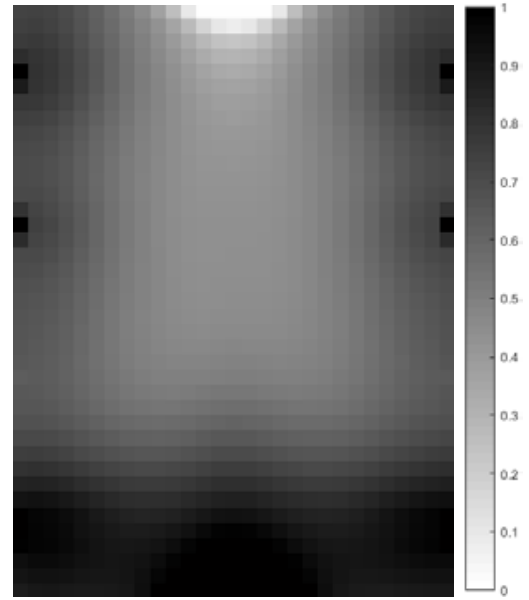

Fig.8. The image reconstructed by the MRR method when $\mathrm{t}=5.9 \mathrm{~h}$

\section{CONCLUSIONS}

The tracking of the migration of ice frontal surface is crucial for the understanding of the underlying physical mechanisms in freezing soil. For the first time, in this paper the ECT method is used to visualize the migration of ice frontal surface in freezing soil. With the main motivation of the improvement of the imaging quality, a loss function with multiple regularizers that incorporate the prior information associated with the imaging objects is proposed to convert the ECT image reconstruction task into an optimization problem with the help of the Tikhonov regularization method. An iteration scheme that integrates the superiority of the SBI method is developed to search for the optimal solution of the proposed loss function. In contrast to common ECT sensors, an unclosed electrodes sensor is designed for satisfying the requirements of practical measurements. An experimental system of one dimensional freezing in frozen soil is constructed and the medium distribution images of the freezing process at different time instants are reconstructed by the proposed reconstruction method. The locations of the migration of ice frontal surface match well with practical process, which validates the feasibility and effectiveness of the ECT visualization method and the proposed reconstruction method. Research findings introduce a promising method for the visualization measurement of the migration of ice frontal surface.

Practical applications indicate that each measurement method has its advantages and disadvantages, and may show different performances to different measurement objects. Future investigations should be implemented to evaluate the accuracy and sensitivity of the method, to develop appropriate reconstruction models and effective numerical methods and to discover optimal sensor structures and discretization method of the reconstruction domain for better measurement results.

\section{REFERENCES}

[1] Zhou, Y.W., Guo, D.X., Cheng, G.D. (2000). Frozen Ground in China. Beijing: Science Press.

[2] Wu, Q.B., Cheng, G.D., Ma, W. (2003). The influence of Qinghai-Tibet railway from the change of permafrost. Science in China, Series D, 33 (12), 115122.

[3] Xu, X.Z., Wang, J.C., Zhang, L.X. (2001). Frozen Soil Physics. Beijing: Science Press.

[4] Liu, Z.L., Zhang, X.P., Li, H.S. (2005). A damage constitutive model for frozen soils under uniaxial compression based on CT dynamic distinguishing. Rock and Soil Mechanics, 26, 542-546.

[5] Zhang, L.X., Pu, Y.B., Liao, Q.R., Gu, Q.R. (1998). Dynamic measurement on the changing process of the moisture and density in freezing soil. Science in China, Series D, 28, 459-462.

[6] Song, L., Liu, T.F., Huang, J.H. (2005). Research on ground penetrating radar (GPR) exploration on artificial frozen wall development. Journal of China University of Mining \& Technology, 34, 143-147.

[7] Delaney, A.J., Peapples, P.R., Arcone, S.A. (2001). Electrical resistivity of frozen and petroleumcontaminated fine-grained soil. Cold Regions Science and Technology, 32, 107-119.

[8] Mercier, O.R., Hunter, M.W., Callaghan, P.T. (2005). Brine diffusion in first-year sea ice measured by Earth's field PGSE-NMR. Cold Regions Science and Technology, 42, 96-105.

[9] Xu, X.Z., Deng, Y.S. (1991). Experiment Research of Moisture Movement in Frozen Soil. Beijing: Science Press.

[10] Xie, C.G., Huang, S.M., Hoyle, B.S., Thorn, R., Lenn, C., Snowden, D., Beck, M.S. (1992). Electrical capacitance for flow imaging: System model for development of image reconstruction algorithms and design of primary sensors. IEE Proceedings G Circuits, Devices and Systems, 139, 89-98.

[11] Warsito, W., Fan, L.S. (2001). Neural network based multi-criterion optimization image reconstruction technique for imaging two-and three-phase flow systems using electrical capacitance tomography. Measurement Science and Technology, 12, 21982210. 
[12] Yang, W.Q., Peng, L.H. (2003). Image reconstruction algorithms for electrical capacitance tomography. Measurement Science and Technology, 14, R1-R13.

[13] Tikhonov, A.N., Arsenin, V.Y. (1977). Solution of IllPosed Problems. New York: V.H. Winston \& Sons.

[14] Landweber, L. (1951). An iteration formula for fredholm integral equations of the first kind. American Journal of Mathematics, 73, 615-624.

[15] Liu, S., Fu, L., Yang, W.Q., Wang, H.G., Jiang, F. (2004). Prior-online iteration for image reconstruction with electrical capacitance tomography. IEE Proceedings - Science, Measurement and Technology, 151, 195-200.

[16] Takei, M. (2006). GVSPM image reconstruction for capacitance CT images of particles in a vertical pipe and comparison with the conventional method. Measurement Science and Technology, 17, 21042112.

[17] Soleimani, M., Lionheart, W.R.B. (2005). Nonlinear image reconstruction for electrical capacitance tomography using experimental data. Measurement Science and Technology, 16, 1987-1996.

[18] Wang, H.X., Tang, L., Cao, Z. (2007). An image reconstruction algorithm based on total variation with adaptive mesh refinement for ECT. Flow Measurement and Instrumentation, 18, 262-267.

[19] Fang, W.F. (2004). A nonlinear image reconstruction algorithm for electrical capacitance tomography. Measurement Science and Technology, 15, 21242132.

[20] Lei, J., Liu, S., Guo, H.H., Li, Z.H., Li, J.T., Han, Z.X. (2011). An image reconstruction algorithm based on the semiparametric model for electrical capacitance tomography. Computers and Mathematics with Applications, 61, 2843-2853.

[21] Ortiz-Aleman, C., Martin, R., Gamio, J.C. (2004). Reconstruction of permittivity images from capacitance tomography data by using very fast simulated annealing. Measurement Science and Technology, 15, 1382-1390.

[22] Banasiak, R., Soleimani, M. (2010). Shape based reconstruction of experimental data in 3D electrical capacitance tomography. NDT \& E International, 43, 241-249.

[23] Cao, Z., Xu, L.J., Fan, W.R., Wang, H.X. (2011). Electrical capacitance tomography for sensors of square cross sections using Calderon's method. IEEE Transactions on Instrumentation and Measurement, 60, 900-907.
[24] Cao, Z., Xu, L.J., Wang, H.X. (2010). Electrical capacitance tomography with a non-circular sensor using the dbar method. Measurement Science and Technology, 21, 1-6.

[25] Watzenig, D., Brandner, M., Steiner, G. (2007). A particle filter approach for tomographic imaging based on different state-space representations. Measurement Science and Technology, 18, 30-40.

[26] Soleimani, M., Vauhkonen, M., Yang, W.Q., Peyton, A., Kim, B.S., Ma, X.D. (2007). Dynamic imaging in electrical capacitance tomography and electromagnetic induction tomography using a Kalman filter. Measurement Science and Technology, 18, 32873294.

[27] Soleimani, M., Mitchell, C.N., Banasiak, R., Wajman, R., Adler, A. (2009). Four-dimensional electrical capacitance tomography imaging using experimental data. Progress in Electromagnetics Research (PIER), 90, 171-186.

[28] Lei, J., Liu, S., Wang, X.Y. (2012). Dynamic inversion in electrical capacitance tomography using the ensemble Kalman filter. IET Science, Measurement and Technology, 6, 63-77.

[29] Rudin, L. Osher, S., Fatemi, E. (1992). Nonlinear total variation based noise removal algorithms. Physica $D$, 60, 259-268.

[30] Goldstein, T., Osher, S. (2009). The split Bregman method for L1-regularized problems. SIAM Journal on Imaging Sciences, 2, 323-343.

[31] Lv, X.G., Song, Y.Z., Wang, S.X., Le, J. (2013). Image restoration with a high-order total variation minimization method. Applied Mathematical Modelling, 37, 8210-8224.

[32] Beck, A., Teboulle, M. (2009). A fast iteration shrinkage-thresholding algorithm for linear inverse problems. SIAM Journal on Imaging Sciences, 2, 183202.

[33] Cai, J.F., Candes, E.J., Shen, Z. (2008). A singular value thresholding algorithm for matrix completion. SIAM Journal on Optimization, 20, 1956-1982.

[34] Wang, H.G., Liu, S., Yang, W.Q., Jiang, F. (2003). The usage of new AC-based capacitance tomography system in experiment. Chinese Journal of Scientific Instrument, 24, 7-10.

Received April 18, 2016 Accepted November 22, 2016. 